\title{
Services in the Long Tail World: Challenges and Opportunities
}

\author{
Neel Sundaresan \\ Sr. Director and Head, eBay Research Labs \\ nsundaresan@ebay.com
}

\begin{abstract}
This talk will focus on Internet based systems that are primarily participatory in nature. In such systems, we need to think beyond infrastructure, data,and algorithms. While these entities are well understood from the service architecture point of view, the demands of participatory systems are different. In a massive-scale system like eBay thats highly participatory in nature, user roles, actions and interactions affect and influence how the system functions and scales. While applications and platforms as service are well understood in the current, evolution through participation mandates the need for additional service orientations. For instance, interface as a service through programmable implementations or user experience as a service through programmable visual elements and interactions can be easily perceived. Machines and machine algorithms will take us part of the way but making them scalable and adaptable to change is a challenge. We need to talk about augmented intelligence where machine power coexists with and is complemented by human intelligence. Designing scalable services and applications in this dynamic context pose interesting challenges and new opportunities. This ltalk will focus on the unique nature of this long tail world.
\end{abstract}

